\title{
IDENTIFICATION AND CHARACTERIZATION OF A UNIQUE RIBOSOMAL FRAMESHIFTING SIGNAL IN SARS-COV ORF3A
}

\author{
Xiao X. Wang, Ying Liao, Sek M. Wong, and Ding X. Liu*
}

\section{INTRODUCTION}

Severe acute respiratory syndrome coronavirus (SARS-CoV) 3a gene encodes a protein of 274 amino acids. Recent studies showed that $3 \mathrm{a}$ is a minor structural protein. ${ }^{1}$ A heterogeneous population of sgRNA 3 transcripts, containing one, two, or three nucleotide insertion in a six $\mathrm{T}$ stretch located 18 nucleotides downstream of the $3 \mathrm{a}$ initiation codon, was identified in SARS-CoV-infected cells as well as in the sera of SARS patients. ${ }^{2}$ Here we report that a $+1 /-1$ frameshifting event occurs in the insertion site. A mechanism of simultaneous slippage at both $\mathrm{P}$ and A sites may account for the frameshifting event.

\section{MATERIALS AND METHODS}

Construction of plasmids: Wild-type SARS 3a cDNA was amplified by PCR and was digested with EcoRV and EcoRI. The digested fragment was cloned into the two sites of pFlag vector, generating $\mathrm{pF}-3 \mathrm{a} / 6 \mathrm{~T}$. Constructs with $7 \mathrm{~T}$ and $8 \mathrm{~T}$ were made by sitedirected mutagenesis.

PCR fragment of EGFP from pEGFP-C1 (Clontech) was digested with BglII and EcoRV and cloned into these two sites on pSARS-3a/7T, generating pEGFP-3a. Deletions in EGFP or 3 a regions were made by overlapping PCR.

In vitro transcription, translation, and transient expression of $3 a$ and its mutants in Cos-7 cells: One microgram of plasmid DNA was transcribed and translated in a TnT coupled in vitro translation system (Promega) in rabbit reticulocyte lysate (RRL). The polypeptides were labeled with ${ }^{35} \mathrm{~S}-$ methionine.

\footnotetext{
* Xiao X. Wang, Sek M. Wong, Ding X. Liu, National University of Singapore, 117543. Ying Liao, Ding X. Liu, Nanyang Technological University, Singapore, 637551. Ding X. Liu, Institute of Molecular and Cell Biology, Singapore, 138673.
} 
SARS-CoV 3a sequences placed under the control of a T7 promoter were transiently expressed in mammalian cells using the recombinant vaccinia virus (vTF7-3) system. In this study, the transfection reagent used was Effecten Transfection Kit (Qiagen).

SDS-polyacrylamide gel electrophoresis (SDS-PAGE) and Western blot: Electrophoresis of viral polypeptides was performed on SDS-12\% polyacrylamide gels. After transferring to PVDF membrane by Semi-dry transfer (Bio-Rad), proteins were detected with $3 \mathrm{a}$ antibody raised in rabbits, anti-FLAG antibody (Sigma), and His-tag monoclonal antibody (Santa Cruz).

\section{RESULTS}

\subsection{A Frameshifting Event Occurs in SARS-CoV ORF3a}

Full-length cDNA covering the $3 \mathrm{a}$ and $3 \mathrm{~b}$ region with six, seven and eight Ts were translated in vitro (Fig. 1a). A band equivalent to the full-length 3 a protein was expressed from all three clones (Fig. 1a, lanes 1-3). To further confirm the 3 a expression in intact cells, pF-3a with a Flag tag at the $\mathrm{N}$ terminal (Fig. 1b) was expressed in Cos-7 cells and detected by Western blot. The 3 a expression was detected in all three clones except in control (Fig. 1b, lanes 1-3). These results suggested that a +1 frameshift in ORF3a with seven Ts and a -1 frameshift in ORF3a with eight Ts occurred during translational elongation in order to maintain the full length ORF3a expression.

\subsection{Identification of the Slippage Site by Mutagenesis Studies}

To characterize the slippage site, point mutations of the seven Ts (from $\mathrm{T}$ to $\mathrm{C}$ ) were made, giving rise to $\mathrm{pF}-3 \mathrm{a} / 7 \mathrm{~T}(\mathrm{M} 1-\mathrm{M} 7)$. In addition, the nucleotide immediately downstream the seven $\mathrm{T}$ (A) was also mutated to $\mathrm{C}$, giving rise to $\mathrm{pF}-3 \mathrm{a} / 7 \mathrm{~T}(\mathrm{M} 8)$. In vitro expression (Fig. 2a) showed that the full-length 3a was expressed in pF-3a/7T (lane 1). Mutation of any of the seven Ts significantly decreased the full-length $3 \mathrm{a}$ expression (lanes 2-8). However, the A to $\mathrm{C}$ mutation did not affect the full-length 3 a production (lane 9).

Similar expression patterns were observed in Cos-7 cells transfected with these constructs (Fig. 2b). The full-length 3a was detected in pF-3a/7T (lane 1) and M8 (lane 9) but not in other mutants (lanes 2-8), confirming that mutation of any $\mathrm{T}$ to $\mathrm{C}$ could greatly reduce the full-length 3 a expression.

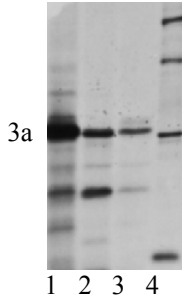

Figure 1a. In vitro expression of 3a/6T (1), 7T (2), and 8T (3). 4: markers.

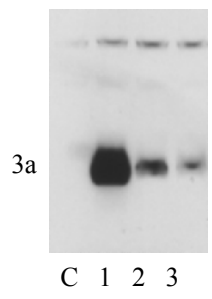

Figure 1b. Expression of $3 \mathrm{a} / 6 \mathrm{~T}$ (1), 7T (2) and 8T (3) in Cos-7 cells. C: mock transfection. 


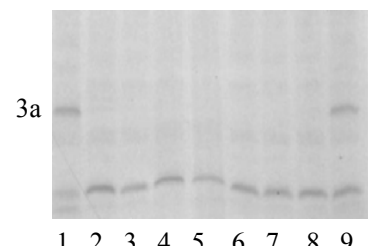

Figure 2a. In vitro expression of ORF3a with wild-type (1) and mutant 7T constructs (2-9).

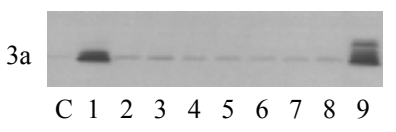

Figure 2b. Expression of ORF3a with wildtype (1) and mutant 7T constructs (2-9) in Cos-7 cells. C: mock transfection.

\subsection{Deletion Analysis of Sequences Upstream of the Slippage Site}

To analyze the effect of the upstream sequences on the frameshifting efficiency, the 5 ' end of $3 \mathrm{a} / 7 \mathrm{~T}$ was fused with the EGFP gene in frame. This extension expected to produce a $28-\mathrm{kDa}$ termination product. The shifted full-length protein (FS) was approximately $55 \mathrm{kDa}$. In vitro translation showed the detection of a major band of 28 $\mathrm{kDa}$ and a minor band of $55 \mathrm{kDa}$ (Fig. 3a, lane 1). The expression of the $55-\mathrm{kDa}$ frameshifting product in intact cells was also detected by Western blot using anti-3a antibody (Fig. 3b, lane 1). Deletions of 63 (pEGFP $\Delta 1-3 a), 123$ (pEGFP $\Delta 2-3 a$ ) and 183 ( $\mathrm{pEGFP} \Delta 1-3 \mathrm{a}$ ) nucleotides, respectively, in the $\mathrm{C}$ terminal region of EGFP were made and expressed both in vitro and in intact cells. No substantial changes of the frameshifting efficiency was observed (Fig. 3a \& 3b, lanes 2-4), indicating that the upstream sequence renders no obvious effect.

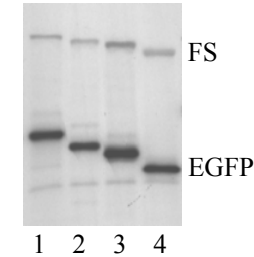

Figure 3a. In vitro expression of pEGFP$3 \mathrm{a} / 7 \mathrm{~T}$ (1) and the 5 , deletion constructs pEGFP $\triangle 1-3 \mathrm{a}(2), \mathrm{pEGFP} \Delta 2-3 \mathrm{a}$ (3), and pEGFP $\triangle 3-3 \mathrm{a}(4)$.

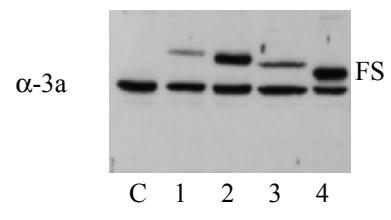

Figure 3b. Expression of pEGFP-3a/7T (1) and the 5 ' deletion constructs pEGFP $\Delta 1-3 \mathrm{a}$ (2), pEGFP $\triangle 2-3 \mathrm{a}(3)$, and $\mathrm{pEGFP} \Delta 3-3 \mathrm{a}(4)$ in $\operatorname{Cos}-7$ cells. C: mock transfection.

\subsection{Deletion Analysis of the Downstream Sequence}

Analysis of the folding of the sequence downstream of the seven Ts showed the presence of two potential stem loops, forming from nucleotides 39 to 56 (starting from AUG for $3 \mathrm{a}$ ) and nucleotides 62 to 88 . The loop regions could partially pair with the downstream sequences and form potential pseudoknot structures. To analyze the effects of these regions on the frameshifting efficiency, five deletion constructs, pEGFP $3 \mathrm{a} \Delta 1$ to $\Delta 5$, were made based on pEGFP-3a/7T (Fig. 4a). In vitro translation of these deletion constructs (Fig. 4b) revealed the detection of both the termination products (EGFP) and the full length frameshifted products (FS). Compared to pEGFP-3a/7T, only $\Delta 4$ showed a slightly decreased detection of the FS product (Fig. 4b, lane 5), while other deletions 


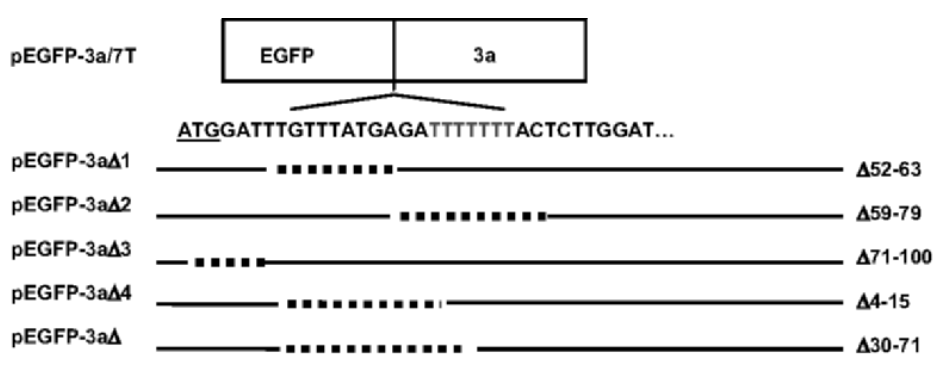

Figure 4a. Diagram of 3a deletions.

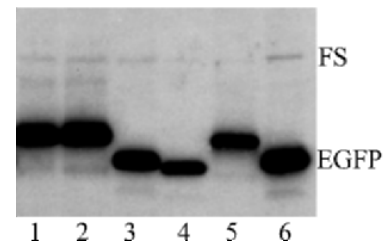

Figure $4 \mathbf{b}$. In vitro expression of EGFP$3 \mathrm{a} / 7 \mathrm{~T}(1)$ and EGFP-3a $\Delta 1$ to 5 (2-6)

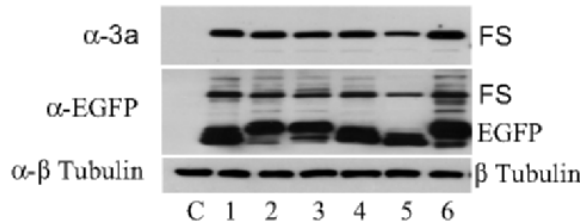

Figure 4c. Expression of EGFP-3a/7T (1) and EGFP-3a $\Delta 1$ to 5 (2-6). C: mock transfection.

didn't have significant effect on the frameshifting efficiency. Similar expression profiles were observed in Cos-7 cells expressing the deletion constructs (Fig. 4c).

\section{DISCUSSION}

In this study, we present evidence demonstrating that expression of full-length $3 \mathrm{a}$ with a single nucleotide insertion required a +1 frameshift at TTT TTT T, characterized as the slippage site. In the event of +1 frameshifting at the slippage site, the PhetRNA $\mathrm{AAA}_{\mathrm{A}}$ could form perfect pair in the +1 frame with TTT. As mutation of any single $T$ at the slippage site significantly impaired the frameshifting efficiency, it suggested that a double slippage mechanism is responsible for this frameshifting event and an exact base pairing is required for the efficient frameshifting. No other stimulators were found for this frameshifting event. This lack of additional stimulators was similar to the previous study with the Herpes simplex virus TK gene. ${ }^{3}$ This may also explain why no frameshifting event was observed when imperfect base-pairing with the shifted frame was introduced.

\section{REFERENCES}

1. Ito, N., Mossel, E. C., Narayanan, K., Popov, V. L., Huang, C., Inoue, T., Peters, C. J., and Makino, S., 2005 , Severe acute respiratory syndrome coronavirus 3a protein is a viral structural protein, J. Virol. 79:3182-3186.

2. Tan, T. H., Barkham, T., Fielding, B. C., Chou, C. F., Shen, S., Lim, S. G., Hong, W., Tan, Y. J., 2005, Genetic lesions within the 3a gene of SARS-CoV, Virol. J. 2:51.

3. Horsburgh, B., Kollmus, H., Hauser, H., and Coen, D., 1996, Translational recoding induced by G-rich mRNA sequences that form unusual structures, Cell 86:949-959. 